\title{
Alternative Axial Distances for Spherical Regions of Central Composite Designs
}

\author{
Linus Ifeanyi Onyishi and F. C. Eze
}

\begin{abstract}
Alternatives to the existing axial distances of the Central Composite Design (CCD) in spherical design using three axial distances were studied. The aim of this study is to determine a better alternative to already existing axial distances whose prediction properties are more stable in the spherical design regions. Using the concepts of the three Pythagorean means, the arithmetic, harmonic and geometric axial distances for spherical regions were developed. The performances of the alternative axial distances were compared with the existing ones using the $D$ and $G$ optimality criteria. The study shows that the alternative axial distances are better using the $D$ and $G$ optimality criteria.
\end{abstract}

\section{Introduction}

Whenever multiple system variables may influence the outputs, response surface methodology (RSM) could be utilized in assessing the relationship between the dependent (response) and independent (input) variables as well as optimize the relevant processes (Mahsa et al. [9] and Diya'uddeen et al. [5]). Response surface methodology (RSM) is a collection of mathematical and statistical techniques for emperical model building. By careful design of experiments, the objective of RSM is to optimize a response (output variable) of interest which is influenced by several independent variables (input variables), Montgomery [10]. In this context, an experiment is a series of tests called runs in which changes are made to the input variables to understand the reasons for the changes in the output variable. In real life application of response surface methodology, the independent variable can be more than one.

Received: May 4, 2020; Accepted: June 5, 2020

2010 Mathematics Subject Classification: 62.

Keywords and phrases: spherical design, optimality criteria, axial distances, central composite design, Pythagorean means. 
The relationship between a response variable of interest, $y$, and the $k$ independent variables is usually described by a first-order response surface model,

$$
y=\beta_{0}+\beta_{i} \sum_{i=1}^{k} x_{i}+e,
$$

where $\beta_{0}$ and $\beta_{i}$ are the coefficients (parameters) of the model, $x_{i}$ 's are the independent variables and $e$ is the random error associated with the response variable such that the random error is normally and independently distributed with zero means and variance, $\sigma_{e}^{2}$; that is, $e \sim N I D\left(0, \sigma_{e}^{2}\right)$. However, if curvature exists, the relationship between the response variable and the independent variables is more appropriately described by a second-order response model,

$$
y_{i j}=\beta_{0}+\sum_{i=1}^{k} \beta_{i} x_{i}+\sum_{i=1}^{k} \beta_{i i} x_{i}^{2}+\sum_{i=1}^{k} \sum_{j=i+1}^{k} \beta_{i j} x_{i} x_{j}+e_{i j},
$$

where $\beta_{0}, \beta_{i}, \beta_{i i}$ and $\beta_{i j}$ are coefficients of the response surface model, other components retain their original meanings. Expressed in matrix form, equation (2) becomes

$$
\mathbf{y}=\beta_{0}+\mathbf{x}^{\prime} \mathbf{b}+\mathbf{x}^{\prime} \mathbf{B} \mathbf{x}+e,
$$

where $\mathbf{y}$ is the $N \times 1$ vector of responses, $\beta_{0}$ is a constant, $\mathbf{x}$ is a point in the design space spanned by the design, $\mathbf{b}$ is a $k \times 1$ vector of first-order (linear) regression coefficients and $N$ is the number of design runs, $\mathbf{B}$ is a $k \times k$ symmetric matrix whose main diagonal entries are the coefficients of the pure quadratic terms and the offdiagonal entries are coefficients of one-half the mixed quadratic (interaction) terms, and $e$ is the random error term associated with the response. There is a total of $p=(k+1)(k+2) / 2$ model parameters to be estimated from the second-order response surface model which includes one constant, $k$ first-order (linear) terms, $k$ quadratic terms and $k(k-1) / 2$ interaction terms. The linear terms are the first-order components of the second-order model while the interaction terms are the cross-products of the secondorder model.

Designs for fitting second-order response surface models like that of equation (2) are 
called second-order response surface designs. However, a second-order response surface design is often chosen on the consideration of several criteria. Myers et al. [11] and Anderson-Cook et al. [2] stated that good second-order designs should:

(a) result in a good fit of the model to the data; (b) provide sufficient information to allow for a test for model lack of fit; (c) allow models of increasing order to be constructed sequentially; (d) provide an estimate of pure experimental error; (e) be insensitive (robust) to the presence of outliers in the data; (f) be robust to errors in control of design levels; ( $\mathrm{g}$ ) be cost effective, that is, do not require too many runs; (h) allow experiments to be performed in blocks; (i) provide a check on the homogenous variance assumption; and (j) provide a good distribution of the variance of the predicted responses throughout the design region.

However Anderson-Cook et al. [2] observed that not all the criteria are required or necessarily important in every RSM application. Some of the criteria listed above also present potential conflict with each other. For instance, one of the criteria suggests small experimental runs for cost effectiveness while another requires good distribution of the prediction variance. Studies have shown that often times, small design runs provide insufficient information for prediction variance evaluation (Li et al. [8]).

Second-order models are used primarily for optimization and therefore, a model that performs well in response prediction is vital. This brings about the importance of the prediction variance highlighted in one of the criteria for adequate evaluation of design performance. Characterization of the prediction variance is very important in comparing competing designs. A good design will have minimum prediction variance distributed throughout the entire design space.

There are types of CCD depending on the choice of $\alpha$ value which is the axial runs from the center which specifies the type of Central Composite Designs. The most commonly used Central Composite Designs are: Spherical Central Composite Design (SCCD), Rotatable Central Composite Design (RCCD), Orthogonal Central Composite Designs (OCCD) and Face Centered Cube Design (FCCD). The choices of these designs depend on the axial runs $(\alpha)$, cube and center points $\left(n_{c}\right)$ (Eze and Ngonadi [6]).

For $\alpha=\sqrt{k}$, we have the spherical $\mathrm{CCD}, \alpha=\sqrt[4]{f}$ gives the rotatable $\mathrm{CCD}$, $\alpha=\sqrt[4]{k}$ gives the practical CCD and $\alpha=1$ gives the Face-centered CCD. The problem with the spherical alpha, as identified by $\mathrm{Li}$ et al. [8], is that as the number of factors 
increases, these alpha values could give impracticable axial distances. For instance, if $k=10$, then $\sqrt{10}=3.1623$ for the spherical which are not feasible values when compared relative to -1 and +1 , extreme values of the cube. Anderson and Whitcomb [1] proposed the practical alpha, $\alpha=\sqrt[4]{k}$ as a compromise for the spherical alpha. The practical alpha, which is less extreme when compared to the spherical also offers reasonable variance inflation factor (VIF) as $k$ increases (Li et al. [8]). However, often times, the practical $\alpha$ tends to locate the star points very close to the centre of the design region that it is difficult to determine the true precision of the designs' prediction capabilities. For instance, in the study by Li et al. [8], despite the advantages posed by the practical axial distance, the CCD with spherical $\alpha$ consistently gave smaller and more stable prediction variance throughout the design region by using fraction of design space plots.

The works of Box and Wilson [4] brought to fore the concepts of spherical $\alpha$ for the CCD. The spherical alpha locates the star points at a distance of $\sqrt{k}$ from the centre of the spherical design space, where $k$ is a positive integer and represents the number of experimental factors. This places the star points and the factorial points on the surface of a sphere of radius, $\sqrt{k}$. The spherical axial distance of the star points usually provide values that are not feasible for large number of experimental factors. For $k=20$, for instance, $\alpha=4.4721$ which is an impractical value considering the fact that the extreme values of the design are placed at -1 and +1 .

The drawback of the spherical and rotatable axial distances with respect to high alpha values when there are high number of factors led to the recommendation of the practical axial distance by Anderson and Whitcomb [1]. The practical axial distance defines the practical CCD and is given by $\alpha=\sqrt[4]{k}$. This axial distance is a compromise between the cuboidal and spherical axial distances, $\alpha=1$ and $\alpha=\sqrt{k}$, respectively. According to the authors, the practical axial distance offers small and stable prediction variance with acceptable variance inflation factors. However, recent studies have shown that despite the obvious advantages of the practical axial distance, it does not necessarily provide consistently small prediction variances throughout the entire design region. In a study by Li et al. [8], the CCD with spherical $\alpha$ is consistently superior to that of the practical $\alpha$ in the spherical regions. 
The aim of the study is therefore to construct alternative axial distances for the spherical design regions of the central composite designs which are less extreme but provides more stable prediction precision for the design.

\section{Methodology}

\subsection{Pythagorean means}

The axial distance for the spherical region is developed using the three classical Pythagorean means namely the arithmetic mean, harmonic mean and geometric means. The Arithmetic mean A of a set of data $a_{1}, a_{2}, \ldots, a_{n}$ is defined as

$$
A=\frac{1}{n} \sum_{i=1}^{n} a_{i}=n^{-1} \sum_{i=1}^{n} a_{i}
$$

Similarly the harmonic mean is expressed as the reciprocal of the arithmetic mean of the reciprocals of the given set of observations $x_{1}, x_{2}, \ldots, x_{n}$ as

$$
H=\frac{n}{\frac{1}{x_{1}}+\frac{1}{x_{2}}+\ldots+\frac{1}{x_{n}}}=\frac{n}{\sum_{i=1}^{n} \frac{1}{x_{i}}}=\left(\frac{\sum_{i=1}^{n} x_{i}^{-1}}{n}\right)^{-1} .
$$

The geometric mean of a data set $\left\{a_{1}, a_{2}, \ldots, a_{n}\right\}$ is given by

$$
\left(\prod_{i=1}^{n} a_{i}\right)^{\frac{1}{n}}=\sqrt[n]{a_{1} a_{2} \ldots a_{n}}
$$

The values of $k$ ranges from 3 to 8 from where the axial distances were developed. The efficiency of these designs was evaluated using $D$ and $G$ efficiency. The highest value of the $D$ and $G$ efficiency is the most efficient to a particular efficiency criteria.

\subsection{Prediction variance}

In the design space $x$, the prediction variance is given by

$$
\operatorname{var}[y(x)]=\sigma^{2} x^{\prime m}\left(X^{\prime} X\right)^{-1} x^{m},
$$


where, $\left(X^{\prime} X\right)$ and $\left(X^{\prime} X\right)^{-1}$ are information matrix and its inverse respectively of the second order composite design. The design matrix is $X$ and $\mathbf{x}^{\mathbf{m}}=\left(1, x_{1}, \ldots, x_{k} ; x_{1}^{2}, \ldots, x_{k}^{2} ; x_{1} x_{2}, \ldots, x_{k-1} x_{k}\right)$ is the vector of design points in the design space. $x_{1}, x_{2}, \ldots, x_{k} ; x_{1}^{2}, x_{2}^{2}, \ldots, x_{k}^{2} ; x_{1} x_{2}, \ldots, x_{k-1} x_{k}$ are the design points into linear, quadratic and mixed components of the model respectively. The Scaled Prediction Variance (SPV) of equation (7) is

$$
\frac{N \operatorname{var} y(x)}{\sigma^{2}}=N x^{\prime m}\left(X^{\prime} X\right)^{-1} x^{m} .
$$

The unknown parameter in equation (8) makes it difficult to use the equation. To eliminate the unknown parameter, Onukogu [12] assume $\sigma^{2}=1$. However, some experimenters prefer the unscaled prediction variance (UPV) for design evaluation given by

$$
\frac{\operatorname{var}[y(x)]}{\sigma^{2}}=x^{\prime m}\left(X^{\prime} X\right)^{-1} x^{m}
$$

In equation (8), the cost of experimentation, $N$ is influenced by using larger designs. On the other hand, scaled prediction variance allows the practitioner to measure the variance of the predicted responses on a per observation basis, thereby penalizing larger designs over smaller ones (Atkinson and Donev [3] and Li et al. [8]). By penalizing larger designs it means the prediction variance of such designs is expected to get bigger when it is scaled (multiplied by $N$ ). The rationale for scaling the prediction variance is to account for the cost of the design, represented by $N$ in comparing designs of various sizes. However, there is increasing awareness for the use of the UPV in design evaluation. Goos [7] and Piepel [13] argue that larger designs often lead to smaller prediction variances and provide the experimenter with more useful information than unscaling the prediction variance.

Among competing designs, the design(s) with the smallest and stable scaled or unscaled prediction variance is/are the most desirable. Stability is in the sense that there is small difference between the minimum and maximum SPV of a design.

\section{3. $D$-efficiency}

A $D$-optimal design maximizes $D$, where $D$ is given by 


$$
D=\operatorname{det}\left[X^{\prime} X\right]
$$

or equivalently minimize $\left|\left(X^{\prime} X\right)\right|^{-1}$, where $X$ is the design matrix.

In summary

$$
\left|X^{\prime} X\right|=\frac{1}{\left|\left(X^{\prime} X\right)^{-1}\right|} \text {. }
$$

\section{4. $G$-efficiency}

$G$-efficiency is based on 'prediction Criterion'. $G$-efficiency or optimality is defined as

$$
\min _{x_{i}, i=1,2, \ldots, n} \operatorname{Max}_{x \in X} \operatorname{var}\left[\left(\hat{y}_{(x)}\right)\right]
$$

This means minimizing the maximum variance over the experimental space of any predicted value. That is

$$
\operatorname{var}\left[\left(\hat{y}_{(x)}\right)\right]=\sigma^{2} f^{\prime}(x)\left(X^{\prime} X\right)^{-1} f(x)
$$

where

$X$ is the design matrix and $x$ is any point in the design region,

$$
f(x)=\left[f_{1}(x), f_{2}(x), \ldots, f_{p}(x)\right]^{\prime} .
$$

The efficiencies of the $D$ and $G$ optimality criteria are determined if the values are exactly 1 or closer to 1 . This means an efficiency of $100 \%$. The $D$ and $G$ efficiencies were calculated using Design Expert software.

\subsection{Axial distances for spherical regions}

The three existing axial distances for the spherical region are, namely: spherical alpha, $\alpha=\sqrt{k}$; practical alpha, $\alpha=\sqrt[4]{k}$ and rotatable alpha, $\alpha=\sqrt[4]{f}$, where $f=2^{k-q}$, $k$ is the number of factors and $q$ is a nonnegative integer less than $k$. The arithmetic, harmonic and geometric means of these three alpha values gave the three new axial distances for the placement of the star points in the spherical region. The first is the arithmetic axial distance. 
Let

$$
A=\frac{1}{n} \sum_{i=1}^{n} a_{i}=n^{-1} \sum_{i=1}^{n} a_{i}
$$

be the arithmetic mean of $n$ random variables with $A$ representing 'arithmetic'. Then the arithmetic mean of the spherical, practical and rotatable axial distances is given by

$$
\alpha n=n^{-1}\left[k^{1 / 2}+k^{1 / 4}+2^{(k-q) / 4}\right], \quad n=3 \text { and } q \geq 0 .
$$

For the harmonic axial distance, let

$$
H=\frac{n}{\frac{1}{x_{1}}+\frac{1}{x_{2}}+\ldots+\frac{1}{x_{n}}}=\frac{n}{\sum_{i=1}^{n} \frac{1}{x_{i}}}=\left(\frac{\sum_{i=1}^{n} x_{i}^{-1}}{n}\right)^{-1}
$$

be the harmonic mean of the $n$ independent random variables. Then the harmonic mean of the three existing axial distances is given by

$$
\begin{aligned}
\alpha_{H n} & =n\left[\frac{1}{\sqrt{k}}+\frac{1}{\sqrt[4]{k}}+\frac{1}{\sqrt[4]{2^{k-q}}}\right]^{-1} \\
& =n\left[k^{-1 / 2}+k^{-1 / 4}+2^{-(k-q) / 4}\right]^{-1}, \quad n=3 \text { and } q \geq 0 .
\end{aligned}
$$

The geometric mean of a set of $n$ independent random variables is

$$
\left(\prod_{i=1}^{n} a_{i}\right)^{\frac{1}{n}}=\sqrt[n]{a_{1} a_{2} \ldots a_{n}}
$$

Hence, the geometric axial distance in the spherical region is

$$
\begin{aligned}
\alpha_{G n} & =\left[\sqrt{k} \times \sqrt[4]{k} \times \sqrt[4]{2^{k-q}}\right]^{\frac{1}{n}} \\
& =\left[k^{\left(\frac{1}{2}+\frac{1}{4}\right)} \times 2^{\frac{k-q}{4}}\right]^{\frac{1}{n}}=\left[\left(2^{\frac{k-q}{4}}\right)\left(k^{\frac{3}{4}}\right)\right]^{\frac{1}{n}} .
\end{aligned}
$$


Therefore,

$$
\alpha_{G n}=\left[k^{3}\left(2^{k-q}\right)\right] \frac{1}{4 n}, \quad n=3 \text { and } q \geq 0 .
$$

Equations (14), (15) and (16) are the alternative axial distances for the CCD in spherical region based on the three Pythagorean means.

\section{Results and Discussions}

The $D$ - and $G$-efficiency values are computed for the six variations of the CCD in spherical region. The variations of the $\mathrm{CCD}$ are based on the various axial distances defining the central composite designs under comparison. The $D$ - and $G$-efficiencies are used to measure any improvement on the performances of the designs by the alternative axial distances. An efficiency of 100 percent is a more preferable design. We considered $k=2$ to 8 factors using three centre points as recommended by Montgomery [10].

The catalogue of the alpha values for the spherical region is shown in Table 1.

Table 1. Catalogue of alpha value for the spherical region.

\begin{tabular}{|l|l|llllll|}
\hline $\boldsymbol{k}$ & $\boldsymbol{f}$ & $\alpha_{S}$ & $\alpha_{P}$ & $\alpha_{R}$ & $\alpha_{A S}$ & $\alpha_{H S}$ & $\alpha_{G S}$ \\
\hline 2 & $2^{2}$ & 1.4142 & 1.1892 & 1.4142 & 1.3392 & 1.3303 & 1.3348 \\
3 & $2^{3}$ & 1.7321 & 1.3161 & 1.6818 & 1.5766 & 1.5530 & 1.5651 \\
4 & $2^{4}$ & 2.0000 & 1.4142 & 2.0000 & 1.8047 & 1.7574 & 1.7818 \\
5 & $2^{5}$ & 2.2361 & 1.4954 & 2.3784 & 2.0366 & 1.9526 & 1.9961 \\
6 & $2^{6}$ & 2.4495 & 1.5651 & 2.8284 & 2.2810 & 2.1417 & 2.2134 \\
& $2^{6-1}$ & 2.4495 & 1.5651 & 2.3784 & 2.1310 & 2.0441 & 2.0891 \\
7 & $2^{7}$ & 2.6458 & 1.6266 & 3.3636 & 2.5453 & 2.3255 & 2.4371 \\
& $2^{7-1}$ & 2.6458 & 1.6266 & 2.8284 & 2.3669 & 2.2283 & 2.3003 \\
& $2^{7-2}$ & 2.6458 & 1.6266 & 2.3784 & 2.2169 & 2.1229 & 2.1712 \\
8 & $2^{8}$ & 2.8284 & 1.6818 & 4.0000 & 2.8367 & 2.5038 & 2.6697 \\
& $2^{8-1}$ & 2.8284 & 1.6818 & 3.3636 & 2.5246 & 2.4088 & 2.5198 \\
& $2^{8-2}$ & 2.8284 & 1.6818 & 2.8284 & 2.4462 & 2.3047 & 2.3784 \\
9 & $2^{9}$ & 3.0000 & 1.7321 & 4.7568 & 3.1630 & 2.6764 & 2.9130 \\
& $2^{9-1}$ & 3.0000 & 1.7321 & 4.0000 & 2.9107 & 2.5847 & 2.7495 \\
\hline
\end{tabular}




\begin{tabular}{|l|l|llllll|}
\hline \multirow{3}{*}{10} & $2^{9-2}$ & 3.0000 & 1.7321 & 3.3636 & 2.6986 & 2.3415 & 2.5952 \\
& $2^{10}$ & 3.1623 & 1.7783 & 5.6569 & 3.5325 & 2.8427 & 3.1686 \\
& $2^{10-1}$ & 3.1623 & 1.7783 & 4.7568 & 3.2325 & 2.7554 & 2.9907 \\
& $2^{10-2}$ & 3.1623 & 1.7783 & 4.0000 & 2.9802 & 2.6583 & 2.8229 \\
& $2^{10-3}$ & 3.1623 & 1.7783 & 3.3636 & 2.7681 & 2.4197 & 2.6644 \\
\hline
\end{tabular}

The values of the $D$ - and $G$-efficiencies values for the axial distances for the spherical regions have been computed and presented in Tables 2 and 3, respectively.

Table 2. $D$-efficiency values with three centre points in spherical region.

\begin{tabular}{|l|l|l|llllll|}
\hline $\boldsymbol{k}$ & $\boldsymbol{F}$ & $\boldsymbol{N}$ & $\alpha_{S}$ & $\alpha_{P}$ & $\alpha_{A S}$ & $\alpha_{H S}$ & $\alpha_{G S}$ & $\alpha_{R}$ \\
\hline 2 & $2^{2}$ & 11 & 61.76 & 44.77 & 57.59 & 57.12 & 57.36 & 61.76 \\
3 & $2^{3}$ & 17 & 70.05 & 52.51 & 62.53 & 53.04 & 53.20 & 100 \\
4 & $2^{4}$ & 27 & 76.40 & 55.80 & 68.98 & 67.30 & 68.16 & 100 \\
5 & $2^{5}$ & 45 & 80.70 & 58.70 & 74.42 & 71.93 & 73.51 & 85.60 \\
6 & $2^{6-1}$ & 47 & 83.50 & 59.60 & 74.62 & 72.33 & 73.51 & 100 \\
7 & $2^{7-1}$ & 81 & 85.94 & 62.16 & 79.41 & 76.29 & 77.91 & 90.61 \\
8 & $2^{8-2}$ & 83 & 87.87 & 63.38 & 79.84 & 76.94 & 78.46 & 87.87 \\
\hline
\end{tabular}

Table 3. $G$-efficiency values with three centre points in spherical region.

\begin{tabular}{|l|l|l|llllll|}
\hline $\boldsymbol{k}$ & $\boldsymbol{F}$ & $\boldsymbol{N}$ & $\alpha_{S}$ & $\alpha_{P}$ & $\alpha_{A S}$ & $\alpha_{H S}$ & $\alpha_{G S}$ & $\alpha_{R}$ \\
\hline 2 & $2^{2}$ & 11 & 87.27 & 57.65 & 83.52 & 83.52 & 83.30 & 61.76 \\
3 & $2^{3}$ & 17 & 89.03 & 79.25 & 85.20 & 79.54 & 79.63 & 77.30 \\
4 & $2^{4}$ & 27 & 95.21 & 58.50 & 92.50 & 91.84 & 92.63 & 76.30 \\
5 & $2^{5}$ & 45 & 86.00 & 80.90 & 88.60 & 89.19 & 88.92 & 83.00 \\
6 & $2^{6-1}$ & 47 & 94.90 & 90.00 & 92.88 & 92.36 & 92.63 & 86.10 \\
7 & $2^{7-1}$ & 81 & 83.68 & 81.64 & 85.37 & 85.52 & 85.46 & 81.06 \\
8 & $2^{8-2}$ & 83 & 94.58 & 95.48 & 97.35 & 96.93 & 97.14 & 94.58 \\
\hline
\end{tabular}


The best results for the $D$-efficiency in spherical region is given by the CCD with rotatable axial distance, $\alpha_{R}$. This is followed by the CCD with spherical axial distances, $\alpha_{S}$. The central composite designs with the three alternative axial distances, $\alpha_{A S}$, $\alpha_{H S}$ and $\alpha_{G S}$, consistently give $D$-efficiency values that are better than the CCD with practical axial distance, $\alpha_{P}$. Among the arithmetic, harmonic and geometric axial distances, the CCD with arithmetic axial distance is the best in terms of $D$-efficiency, followed by the CCD with geometric axial distance. The CCD with harmonic axial distance consistently gave the worst $D$-efficiency performance among the three alternative axial distances. Therefore, the central composite designs with arithmetic, harmonic and geometric axial distances should be used in place of the CCD with practical axial distance when the $D$-efficiency is the criterion chosen by the experimenter for response surface exploration.

The CCDs with arithmetic, harmonic and geometric axial distances, $\alpha_{A S}, \alpha_{H S}$ and $\alpha_{G S}$, gave the highest $G$-efficiency values for $k=5,7$ and 8 factors. Only the $G$-efficiency values of the CCD with spherical axial distance, $\alpha_{S}$, are higher than those of the arithmetic, harmonic and geometric axial distances for $k=3,4$ and 6 factors. In the spherical region, the CCD with practical axial distance offered the lowest $G$-efficiency values, followed by the CCD with rotatable axial distance. It is only at $k=8$ that the CCD with rotatable and spherical axial distances have the same $G$-efficiency values which are however, lower than those of the three new alternative axial distances. Hence, if the $G$-efficiency is the experimenter's preferred criterion for response surface exploration in spherical region, the CCD with arithmetic axial distance should be used or any of harmonic and geometric axial distances. It is only for $k=3,4$ and 6 factors that the CCD with spherical axial distance should be preferred.

\section{Conclusion}

The Pythagorean means of arithmetic, harmonic and geometric alphas were developed as alternative distances for the spherical regions of the central composite design. These axial distances were evaluated using the $D$ and $G$ efficiencies criteria. It was observed that the $D$-efficiency values of the arithmetic, harmonic and geometric axial distances are consistently better than those of the practical axial distance. The $G$-efficiency values of the three alternative axial distances in most cases remained the 
highest among the six variations of the central composite designs simultaneously evaluated in the spherical regions.

\section{References}

[1] M. Anderson and P. Whitcomb, RSM Simplified: Optimizing Processes Using Response Surface Methods for Design of Experiments, Productivity Press, New York, NY, 2005. https://doi.org/10.4324/9781482293777

[2] C. M. Anderson-Cook, C. M. Borror and D. C. Montgomery, Response surface design evaluation and comparison, Journal of Statistical Planning and Inference 139 (2009), 629-641. https://doi.org/10.1016/j.jspi.2008.04.004.

[3] A. C. Atkinson and A. N. Donev, Optimum Experimental Designs, Oxford University Press, New York, 1992.

[4] G. E. P. Box and K. B. Wilson, On the experimental attainment of optimum conditions, Journal of the Royal Statistical Society Series B 13 (1951), 1-38.

https://doi.org/10.1111/j.2517-6161.1951.tb00067.x

[5] B. H. Diya'uddeen, A. R. Abdul Aziz and W. M. A. W. Daud, On the limitation of Fenton oxidation operational parameters: a review, International Journal of Chemical Reactor Engineering 10 (2012), 1-12. https://doi.org/10.1515/1542-6580.2913

[6] F. C. Eze and L. O Ngonadi, Alphabetic optimality criteria for 2k central composite design, Academic Journal of Applied Mathematical Sciences 4(9) (2018), 107-118.

[7] P. Goos, Discussion of "Response surface design evaluation and comparison", Journal of Statistical Planning and Inference 139 (2009), 657-659. https://doi.org/10.1016/j.jspi.2008.04.012.

[8] J. Li, L. Li, C. M. Borror, C. Anderson-Cook and D. C. Montgomery, Graphical summaries to compare prediction variance performance for variations of the central composite design for 6 to 10 factors, Quality Technology and Quantitative Management 6(4) (2009), 433-449. https://doi.org/10.1080/16843703.2009.11673209

[9] A. Mahsa, B. Morteza, N. Sirous and N. A. Abdolhosein, Central composite design for the optimization of the removal of the azo-dye, methyl orange from waste water using the fenton reaction, Journal of Serbian Chemical Society 77(2) (2012), 235-246. https://doi.org/10.2298/JSC110315165A

[10] D. C. Montgomery, Design and Analysis of Experiments, 8th ed., John Wiley and Sons, Inc.. N.Y., 2013. 
[11] R. H. Myers, D. C. Montgomery and C. M. Anderson-Cook, Response Surface Methodology: Process and Product Optimization Using Designed Experiments, 3rd Edition, Wiley and Sons, Inc., New York, N.Y., 2009.

[12] I. B. Onukogu, Foundations of Optimum Exploration of Response Surfaces, Ephrata Press, Nsukka, 1997.

[13] G. F. Piepel, Discussion of "Response surface design evaluation \& comparison" by C. M. Anderson-Cook, C. M. Borror, and D. C. Montgomery, Journal of Statistical Planning and Inference 139 (2009), 653-656. https://doi.org/10.1016/j.jspi.2008.04.008

Linus Ifeanyi Onyishi

Department of Mathematics and Statistics, Federal Polytechnic,

Nasarawa, Nasarawa State, Nigeria

F. C. Eze

Department of Statistics, Nnamdi-Azikiwe University, Awka, Nigeria e-mail: fc.eze@unizik.edu.ng

This is an open access article distributed under the terms of the Creative Commons Attribution License (http://creativecommons.org/licenses/by/4.0/), which permits unrestricted, use, distribution and reproduction in any medium, or format for any purpose, even commercially provided the work is properly cited. 